\title{
The Extraction of Linalool from Coriander Seeds by Steam Distillation
}

\section{Yuelin Zhang, Zhanrong Sun, Xu Chen, Qin Chen*}

School of Life Science, Shanghai University, Shanghai, China

\section{Email address:}

moonlighty114@163.com (Yuelin Zhang),2497195015@qq.com (Zhanrong Sun), xuchen@staff.shu.edu.cn (Xu Chen), chenqincc@staff.shu.edu.cn (Qin Chen)

${ }^{*}$ Corresponding author

\section{To cite this article:}

Yuelin Zhang, Zhanrong Sun, Xu Chen, Qin Chen. The Extraction of Linalool from Coriander Seeds by Steam Distillation. Science Discovery. Vol. 4, No. 2, 2016, pp. 75-78. doi: 10.11648/j.sd.20160402.14

Received: January 12, 2016; Accepted: January 26, 2016; Published: May 4, 2016

\begin{abstract}
Linalool is crucial chemical raw material which is widely used in food flavoring, perfumery cosmetic and pharmaceutical industry. The natural linalool from coriander seeds volatile oil was extracted by steam distillation method and analyzed by Gas Chromatography-Mass Spectrometry (GC-MS). Effects of pretreatment (smash, testa chipping, soak and boiling), solid-liquid ratio (weight of coriander seeds $(\mathrm{g})$ : volume of deionized water $(\mathrm{mL})$ ) and the extractant (ethyl acetate and ether) on natural linalool extracted from the volatile oil were studied. The result shows that when the coriander seeds were smashed and soaked for 3 hours, the ratio of solid-liquid was 1:7, the time of distillation was $4 \mathrm{~h}$ and the extractant was ether, natural linalool of the volatile oil was higher than the others, and the content was about $13.45 \mathrm{~g} / \mathrm{kg}$. These results lay a foundation for the extraction of linalool from coriander seeds by steam distillation.
\end{abstract}

Keywords: Coriander Seed, Linalool, Steam Distillation, GC-MS

\section{水蒸气蒸馏法提取芫荽籽中芳樟醇}

\section{张岳琳, 孙湛蓉, 陈旭, 陈沈 ${ }^{*}$}

生命科学学院, 上海大学, 上海, 中国

\section{邮箱}

moonlighty114@163. com（张岳琳）, 2497195015@qq. com（孙湛蓉）, xuchen@staff. shu. edu. cn（陈旭）, chenqincc@staff. shu. edu. cn (陈沈)

\begin{abstract}
摘要: 芳樟醇作为化工原料, 广泛用于食品、日化调香以及医药等行业。本文以芫荌籽为材料, 采用水蒸汽蒸馏法提 取含有天然芳樟醇的芫荌籽挥发油, 用GC-MS对天然芳樟醇进行定量分析, 探讨预处理方式、料液比、萃取溶剂等对芫 荌籽挥发油中芳樟醇得率的影响。结果表明, 将芫荌籽粉碎、去离子水浸泡 $3 \mathrm{~h}$ 、以料液比为 $1: 7(\mathrm{~m}: \mathrm{V})$ 、水蒸气蒸馏 $4 \mathrm{~h}$ 后, 用乙醚作为萃取溶剂, 此时得率最高, 该条件下芫萎籽中芳樟醇的得率可以达到 $13.45 \mathrm{~g} / \mathrm{kg}$ 。本研究为运用水 蒸气蒸馏法提取植物组织中的芳樟醇奠定了基础。
\end{abstract}

关键词: 芫荌籽, 芳樟醇, 水蒸汽蒸馏, 气相色谱-质谱联用法 


\section{1. 引言}

芳樟醇属于链状萜烯醇类, 具有铃兰花香气 [1]，被广 泛应用于食用香料、化妀品香料及医药领域。当今世界使 用的芳樟醇 $90 \%$ 以上来自 “合成芳樟醇” , 但在合成芳樟醇 的工业生产中, 会产生大量的废气、废液和废渣, 严重污 染环境。虽然含有芳樟醇的天然香料较多, 但可用于大量 提取芳樟醇的天然香料品种少, 导致天然芳樟醇相比较于 合成芳樟醇出现供不应求的现象 [2]。从天然植物中提取并 获得芳樟醇成为研究热点, 芫荽籽中含有脂肪烃、单萜及 其含氧衍生物, 其中含量较高的是芳樟醇 [3]。研究表明, 芫荌籽挥发油中 $70 \%$ 左右为芳樟醇 $[4,5]$, 因此芫荽籽是一 种较好的潜在提取天然芳樟醇的植物组织来源。

常用水蒸汽蒸馏法作为提取植物组织中的挥发油的 方法 [6-8]。水蒸汽蒸馏法 (Hydrodistillation, HD) 是利 用植物精油各组分蒸汽压力的不同利用水蒸汽将精油从 原料中提取出来的方法。该法适合于具有挥发性、能随水 蒸汽蒸出而不被破坏、与水不发生反应、又难溶于水的有 效成分的提取 [9]。目前已报道该方法用于提取金刺梨挥 发油、薰衣草精油及牡丹精油等 $[10,11]$ 。然而, 鲜见用 此方法提取芫荽䊏挥发油实验条件优化的报道, 故本研究 拟以芫荌籽为原料, 结合气相色谱一质谱联用方法, 定量 分析挥发油中的天然芳樟醇。通过对芳樟醇提取工艺的优 化, 为高效提取芫荌籽中的芳樟醇提供试验依据。

\section{2. 材料与方法}

\section{1. 材料与试剂}

芫荌籽：上海瑞奇种业有限公司;

乙醚、乙酸乙酯: 分析纯, 国药集团化学试剂有限公司; 芳樟醇标准品: 上海科佳药检器材有限公司。

\section{2. 主要仪器设备}

调温电热器: $Q / 320683 A A F A 02-2005$, 上海苏进仪器 设备厂;

高速多功能粉碎机: Q-100A3型, 上海冰都电器有限 公司;

气相色谱一质谱联用仪: GCMS-QP2010ULTRA型, 日本 岛津 (Shimadzu) 公司;

色谱柱: Rtx-50色谱柱 $(30.0 \mathrm{~m} \times 0.25 \mathrm{~mm} \times 0.25 \mu \mathrm{m})$, 美国Restek公司。

\section{3. 方法}

\subsection{1. 芫荽籽挥发油的提取}

将经不同预处理后的种子置于圆底三口烧瓶中, 按一 定料液比加入去离子水进行水蒸汽蒸馏(微沸 $4 \mathrm{~h}$ ), 萃取, 将萃取后液体稀释 100 倍放入棕色瓶中常温保存备用。

\subsubsection{GC-MS检测}

色谱及FID条件参照王祖翔、商敬敏等 [12-14] 的文献 略加修改, 具体步骤如下:
色谱条件：升温程序： $80^{\circ} \mathrm{C}$ 保持 $1 \mathrm{~min}$, 以 $6^{\circ} \mathrm{C} / \mathrm{min}$ 升 至 $180^{\circ} \mathrm{C}$, 保持 $2 \mathrm{~min}$, 再以 $10^{\circ} \mathrm{C} / \mathrm{min}$ 升至 $220^{\circ} \mathrm{C}$, 保持 $8 \mathrm{~min}$; 柱箱温度 $80^{\circ} \mathrm{C}$; 进样口温度 $150^{\circ} \mathrm{C}$; 载气为氦气; 分流进 样, 进样量为 $1 \mu \mathrm{L}$ 。

氢火焰离子化检测器 (FID) : 检测器温度 $250^{\circ} \mathrm{C}$; 尾 吹气为氮气/ 空气; 氢气流量 $40 \mathrm{~mL} / \mathrm{min}$; 空气流量 $400 \mathrm{~mL} / \mathrm{min}$; 进样速率 $0.04 \mathrm{sec}$; 停止时间 $2 \mathrm{~min}$ 。

标准曲线的绘制: 将芳樟醇标准样品用乙酸乙酯分别 稀释为 $1.0 \times 10^{-2} \mathrm{~mL} / \mathrm{L}, 1.5 \times 10^{-2} \mathrm{~mL} / \mathrm{L}, 2.0 \times 10^{-2} \mathrm{~mL} / \mathrm{L}$, $2.5 \times 10^{-2} \mathrm{~mL} / \mathrm{L} ， \quad 3.0 \times 10^{-2} \mathrm{~mL} / \mathrm{L} ， \quad 3.5 \times 10^{-2} \mathrm{~mL} / \mathrm{L}$ ， $4.0 \times 10^{-2} \mathrm{~mL} / \mathrm{L} 7$ 个浓度梯度, 用 GC-MS检测, 根据峰面积与 待测物的量成正比的原理, 运用GCMSPostrumAnalysis软 件绘制标准曲线。

\subsection{3. 芳樟醇得率的计算}

芫荌籽的重量记作 $M(\mathrm{~g})$, 萃取剂萃取后提取液的体积 为 $V(\mathrm{~L})$, 芳樟醇标准曲线可计算得出浓度记作 $c(\mathrm{~mL} / \mathrm{L})$, 芳樟醇密度为 $0.861 \mathrm{~g} / \mathrm{mL}$, 提取得到的芳樟醇质量为:

$$
m=0.861 \mathrm{~g} / m L \times V \times c
$$

天然芳樟醇得率为:

$$
P=\frac{m}{M} \times 100 \%
$$

\subsection{4. 不同条件对芳樟醇得率的影响}

分别用乙醚和乙酸乙酯作为萃取剂, 对料液比为 $1: 5$, 预处理为煮沸破壳的芫荌䊏馏出液进行萃取。通过 GC-MS 检测芫荽籽挥发油中天然芳樟醇的得率。

预处理方法分别为粉碎浸泡 $3 \mathrm{~h}$ (用高速多功能粉碎机 将芫荽籽粉碎, 再过 40 目篮后浸泡) 、粉碎煮沸 $1 \mathrm{~h}$ (用高 速多功能粉碎机将芫荽籽粉碎, 再过 40 目篮后浸泡) 、破 壳浸泡 $3 \mathrm{~h}$ 和破壳煮沸 $3 \mathrm{~h}$, 以料液比为 $1: 5(\mathrm{~m}: \mathrm{V})$ 、水蒸气 蒸馏 $4 \mathrm{~h}$ 、乙醚为萃取溶剂。

料液比指的是芫荌籽的质量 $(\mathrm{g})$ 与蒸馏水体积 $(\mathrm{mL})$ 的比值。参照陈红英 [15] 等文献, 以粉碎浸泡 $3 \mathrm{~h}$ 为预处 理, 分别控制料液比为 $1: 15$ (36. $0 \mathrm{~g}$ 芫荌籽: $180 \mathrm{~mL}$ 去离子 水)、 $1: 7$ (25.7g芫荌籽: $180 \mathrm{~mL}$ 去离子水) 、 $1: 9(20.0 \mathrm{~g}$ 芫荌籽: $180 \mathrm{~mL}$ 去离子水), 乙醚为萃取溶剂提取芫荽籽 挥发油。

将芫荽籽进行水蒸气蒸馏, 从第一滴馏出液流出开 始, 取样 $1 \mathrm{~mL}$ 于 $5 \mathrm{~mL}$ 的Eppendorf离心管中, 记作 “ CK” 。 之后每隔 $1 \mathrm{~h}$ 取样 $1 \mathrm{~mL}$ 于 $5 \mathrm{~mL}$ 的Eppendorf离心管中, 蒸馏 $4 \mathrm{~h}$, 依次记作 “ $1 \mathrm{~h} 、 2 \mathrm{~h}$ 、 $3 \mathrm{~h}$ 、 $4 \mathrm{~h}$ ” ，共得到 5 管馏出液（CK、 $1 h 、 2 h 、 3 h 、 4 h)$, 测定不同蒸馏时间下芳樟醇的含量 变化。

\section{3. 结果与讨论}

\section{1. 芳樟醇的定性及定量分析}

采用保留时间和MS/SCAN方法来定性, 利用芳樟醇标 准品出峰时间和 3 个特征离子用于芳樟醇的定性分析。 
标样和样品溶液同时上机测定。选择特征离子为: $\mathrm{m} / \mathrm{z}$ （71、93、121）, 采用外标法进行定量计算。其中前两
个特征离子为芳樟醇标准数据库定性时采用, 最后一个特 征离子用于定量计算。标准品离子图见图1。

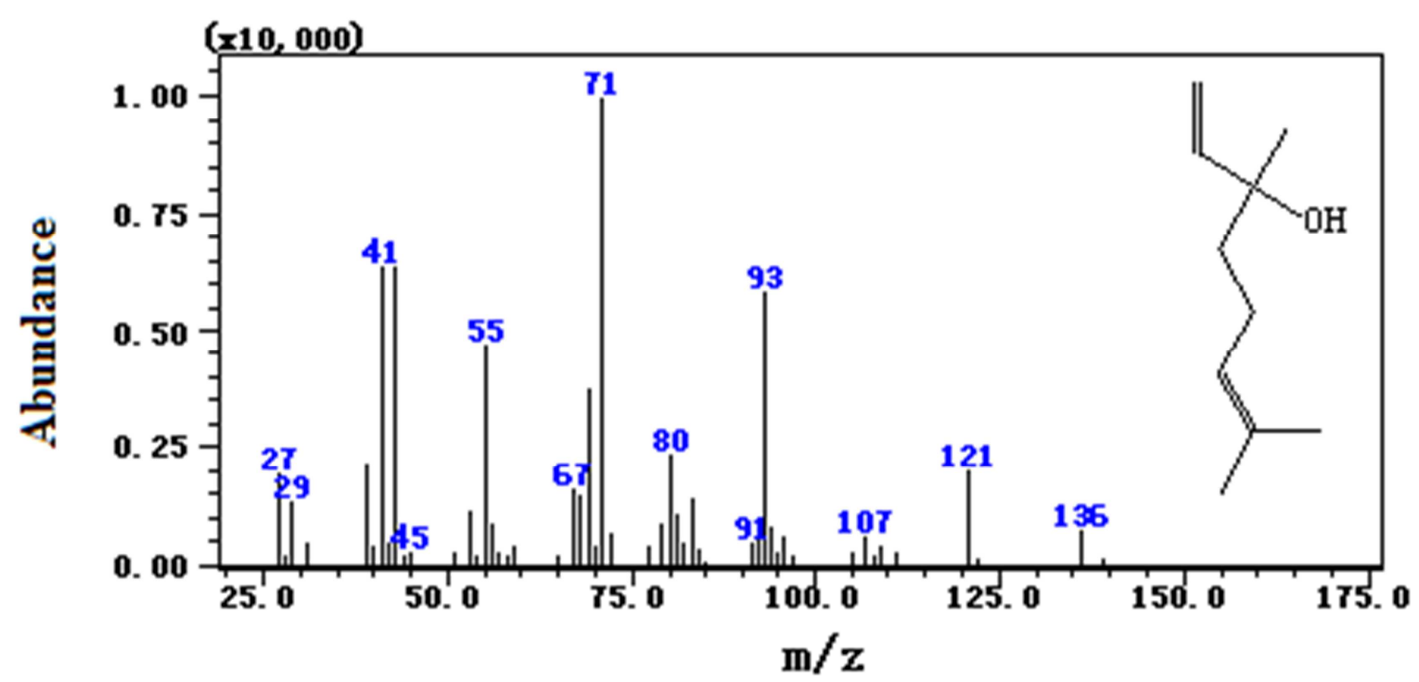

图1 芳樟醇总离子图。

\section{2. 标准曲线的绘制}

选取稀释到不同浓度的芳樟醇标准品, 用 GC-MS测定 其得率, 通过GCMSPostrumAnalysis 软件绘制芳樟醇标准 曲线并计算得出方程: $Y=9597.914 X-5566.302$, 其中 $\mathrm{R}^{2}=0.990$, 线性范围为 $1 \times 10^{-2} \sim 4 \times 10^{-2} \mathrm{~mL} / \mathrm{L}$ 。所得标准曲 线线性良好, 可用于后续芳樟醇得率的分析。

\section{3. 不同条件对芳樟醇得率的影响}

\section{3. 1. 萃取剂对芳樟醇得率的影响}

为了提高天然芳樟醇的得率, 选择高效萃取剂是关键。 萃取剂的萃取效果一般与两个因素有关: 一方面, 萃取剂 与产物之间的溶解度越大, 萃取效果越好; 另一方面, 萃 取剂与溶有产物的溶剂之间的溶解度也会影响萃取效果, 溶解度越大, 萃取效果越差。分别用乙酸乙酯和乙醚作为 萃取溶剂提取后, 用GC-MS测天然芳樟醇得率, 结果列于 表 1 : 以乙酸乙酯作为萃取溶剂所得芳樟醇的得率为 $5.03 \mathrm{~g} / \mathrm{kg}$, 以乙醚作为萃取溶剂所得芳樟醇的得率为 $5.50 \mathrm{~g} / \mathrm{kg}$ 。

在预处理相同、料液比相同的条件下，萃取剂为乙醚 的天然芳樟醇得率, 比萃取剂为乙酸乙酯的天然芳樟醇得 率高。究其原因, 作为常用的萃取剂乙酸乙酯 $20^{\circ} \mathrm{C}$ 时, 溶 解度为 $8.3 \%$; 相比于乙酸乙酯, 乙醚化学性质比较稳定, 很少与除酸之外的试剂反应, 是常用的优良溶剂, $20^{\circ} \mathrm{C}$ 时, 溶解度为 $6.9 \%$, 比乙酸乙酯的溶解度低 $1.4 \%$, 即萃取时, 乙醚更易与水分离, 结果可提取到更多天然芳樟醇, 因此, 本实验最终选择乙醚为萃取剂。

表1 萃取剂对芳樟醇得率的影响。

\begin{tabular}{lll}
\hline 萃取剂 & 芳樟醇得率 $(\mathrm{g} / \mathrm{kg})$ & 显著性分析 $(\mathrm{P}<0.05)$ \\
\hline 乙酸乙酯 & 5.03 & $\mathrm{a}$ \\
乙醚 & 5.50 & $\mathrm{~b}$ \\
\hline
\end{tabular}

\section{3.2. 预处理对天然芳樟醇得率的影响}

芫荽籽经不同预处理后在料液比为 $1: 5$ 、萃取溶剂为 乙醚的情况下分别提取芫荽籽挥发油, 用GC-MS色谱图结 合标准曲线, 测量天然芳樟醇得率, 结果如表2所示。

由表可知, 不同预处理都可提取得到天然芳樟醇, 进 一步比较得出芫荽籽经粉碎后浸泡再蒸馏天然芳樟醇得 率比其他三种预处理高近 2 倍, 而其它预处理组合之间差 异不显著。可能是因为芫荽籽成熟果实坚硬, 用植物组织 粉碎机进行粉碎处理比简单的破壳更好, 煮沸虽然可以除 去芫荌䊏挥发油中低沸点物质, 但会使部分芳樟醇随温度 升高导致分子运动加快 [16], 挥发到空气中。比较这4种 处理方法, 粉碎浸泡法得到芳樟醇的得率最高为 $10.21 \mathrm{~g} / \mathrm{kg}$, 最终选择粉碎浸泡为预处理条件。

表2 预处理对芳樟醇得率的影响。

\begin{tabular}{lll}
\hline 预处理 & 芳樟醇得率 $(\mathrm{g} / \mathrm{kg})$ & 显著性分析 $(\mathrm{P}<0.05)$ \\
\hline 粉碎浸泡 & 10.21 & $\mathrm{a}$ \\
破壳浸泡 & 4.94 & $\mathrm{c}$ \\
破壳煮沸 & 5.49 & $\mathrm{~b}$ \\
粉碎煮沸 & 5.32 & $\mathrm{bc}$ \\
\hline
\end{tabular}

\section{3. 3. 料液比对天然芳樟醇得率的影响}

在水蒸汽蒸馏法提取植物挥发油的过程中, 料液比过 大, 影响挥发油在水中的扩散作用; 过小, 挥发油在水中 的溶解量增大, 影响出油率, 同时能耗增大, 造成浪费。 本试验中分别设置不同料液比 $(1: 5 、 1: 7 、 1: 9)$ 提取芫 荌䊏挥发油, 测得天然芳樟醇得率得表 3 。

由表 3 可知, 在预处理相同、萃取溶剂相同的条件下, 料液比为 $1: 7$ 的天然芳樟醇得率分别是料液比为 $1: 5$ 和 $1: 9$ 的1.32倍和1.44倍。因此, 实验中选取的料液比为 $1: 7$ 。 
表3 料液比对芳樟醇得率的影响。

\begin{tabular}{lll}
\hline 料液比 & 芳樟醇得率 $(\mathrm{g} / \mathrm{kg})$ & 显著性分析 $(\mathrm{P}<0.05)$ \\
\hline $1: 5$ & 10.21 & $\mathrm{a}$ \\
$1: 7$ & 13.45 & $\mathrm{~b}$ \\
$1: 9$ & 9.36 & $\mathrm{a}$ \\
\hline
\end{tabular}

\section{3. 4. 蒸馏时间对天然芳樟醇浓度影响}

水蒸汽蒸馏的时间直接影响产物得率, 时间短, 芫 荌䊏组织中的芳樟醇不能全部扩散到去离子水中; 增加 蒸馏时间, 更多的芳樟醇会扩散到去离子水中, 挥发油 中芳樟醇得率逐渐增加。但蒸馏时间过久, 会使芫荽籽 挥发油加速挥发反而降低天然芳樟醇的得率, 且增加试 验成本, 浪费能源。蒸馏开始后, 收集不同时间段馏出 液, 用 GC-MS检测馏出液中天然芳樟醇的浓度随时间变化 曲线图, 得表4。

由表4可知，不同收集时间，芫荌籽挥发油中天然芳 樟醇含量不同。计算挥发油中天然芳樟醇的含量, 可知, 蒸馏过程中随时间的延长, 芫荽籽挥发油持续流出, 得到 的天然芳樟醇得率显著增加。当蒸馏时间 $4 \mathrm{~h}$ 时, 得率已达 $2.86 \mathrm{~g} / \mathrm{kg}$, 是蒸馏开始时所得天然芳樟醇浓度的 1.6 倍。 最终选择蒸馏时间为 $4 \mathrm{~h}$ 。

表4 蒸馏时间对芳樟醇得率的影响。

\begin{tabular}{llllll}
\hline 样品名称 & CK & 1h & 2h & 3h & 4h \\
\hline 芳樟醇得率 $(\mathrm{g} / \mathrm{kg})$ & 1.72 & 1.97 & 2.49 & 2.28 & 2.86 \\
显著性分析 $(\mathrm{P}<0.05)$ & $\mathrm{a}$ & $\mathrm{b}$ & $\mathrm{d}$ & $\mathrm{c}$ & $\mathrm{e}$ \\
\hline
\end{tabular}

\section{4. 结论}

本试验成功建立了提取芫荌籽挥发油并测定挥发油 中天然芳樟醇的方法, 具体步骤如下: 将芫荌籽用高速多 功能粉碎机粉碎、去离子水浸泡 $3 \mathrm{~h}$ 后, 以料液比为 $1: 7$, 水蒸汽蒸馏 $4 \mathrm{~h}$, 再以乙醚为萃取溶剂, 提取挥发油, 经 GC-MS检测挥发油中天然芳樟醇的得率约为 $13.45 \mathrm{~g} / \mathrm{kg}$, 即 $1.35 \%$ 。文献报道的提取结果分别为 $0.60 \%[3] 、 0.92 \%[17]$ 和 $0.37 \%$ [18], 均低于本试验结果。但本试验仍存在不足, 如所得到的天然芳樟醇是溶于挥发油中, 未分离单体天然 芳樟醇; 同时, 并未对所提取到的芫荽籽挥发油的其他成 分进行定性和定量的检测, 今后将围绕这两个方面展开更 全面的研究。

\section{致谢}

本文为国家大学生创新创业训练计划《䒕荽中天然芳 樟醇的高效提取》(CXGJ-13-040) 的阶段性成果之一。

\section{参考文献}

[1] 路喆, 王朴, 蒋新明, 等. 新疆不同品种的薰衣草精油成分及 得率研究 [J]. 安徽农业科学, 2013, 41(4): 1736 1737, 1739。

[2] 林翔云. 天然芳樟醇与合成芳樟醇 [J]. 化学工程与装 备, 2008, 7:21 26。

［3］李锋, 解成喜, 范维刚, 等. 气相色谱一质谱法分析芫萎籽挥 发油化学成分 $[J]$. 质谱学报, 2005, 26 (2) : 105 107。

[4] 李伟, 封丹, 陆占国. 黑龙江产芫荽籽精油成分及其抗菌活 性 [J]. 中国调味品, 2008, 1:42 45。

[5] 张建民, 宋育航, 王甲林. 芫荽子挥发油中芳樟醇含量测定 [J]. 现代中药研究与实践, 2004, 18 (6) : 44 46。

[6] 曹少华, 刘晓棠, 初探薰衣草精油的提取 [J]. 生物学通 报, 2012, 47 (4) :44〜45。

[7] 张秋霞, 陈计峦, 江英. 薰衣草精油的提取工艺研究 $[J]$. 食 品科技, $2007,5: 123 \sim 125$ 。

[8］刘晓丽, 钟少枢, 于鸿鹏, 等. 微波法和水蒸气蒸馏法提取丁 香精油的研究 [J]. 食品与机械, 2012, 28 (4) : 110 116。

[9] 杨君, 张献忠, 高宏建, 等. 天然植物精油提取方法研究进展 [J]. 中国食物与营养, 2012, 18 (9) : 31-35。

[10] 钟漫, 张晓娟, 杨涛等. 金刺梨挥发油水蒸气蒸馏法工艺优 化研究 $[J]$. 贵州林业科技, 2015, 43(3) : 15 218 。

[11] 李双, 王成忠, 唐晓璇等. 不同提取方法对牡丹精油理化性 质和成分的影响 $[J]$. 食品工业, 2015, 36 (7) : 170 174 。

[12] 李金玉. 利用气相色谱一质谱联用技术测定燕麦中脂肪酸的 组成 $[J]$. 食品与机械, 2011, 27 (3) : 82 83。

[13] 王祖翔, 周洪斌, 蒋俊, 等. 气相色谱-串联质谱法测定复合 调味料中的微量苯甲酸 $[J]$. 食品与机械, 2011, 27 (3) : 84 87 。

[14] 商敬敏, 牟京霞, 刘建民, 等. GC-MS法分析不同产地酿酒葡 萄的香气成分 $[J]$. 食品与机械, 2011, 27 (5): 52 57。

[15] 陈红英, 郭巧玲, 姚龙珠. 水蒸气蒸馏法提取橙皮精油的影 响因素研究 [J]. 中国药业, 2011, 20(1):39 40。

[16] 孙宏. 孜然精油提取工艺的探讨 [J]. 齐齐哈尔大学学 报. 2008, 24(2):34 36。

[17] 李锦兰, 阮星海. 芫荽子挥发油化学成分和致突变性研究 [J]. 食品科学, 1996, 17 (5) : 43 46。

[18］陆占国, 封丹, 李伟. 黑龙江产芫荽籽精油成分及其清楚 $\mathrm{DPPH}$ 自由基能力研究 $[\mathrm{J}]$. 食品与发酵工 业, 2008, 34(1):31 34。 